\title{
Study protocol of the Asian XELIRI
}

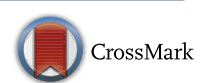
ProjecT (AXEPT): a multinational, randomized, non-inferiority, phase III trial of second-line chemotherapy for metastatic colorectal cancer, comparing the efficacy and safety of XELIRI with or without bevacizumab versus FOLFIRI with or without bevacizumab

Masahito Kotaka', Ruihua Xu², Kei Muro ${ }^{3}$, Young Suk Park ${ }^{4}$, Satoshi Morita ${ }^{5}$, Satoru Iwasa ${ }^{6}$, Hiroyuki Uetake ${ }^{7}$, Tomohiro Nishina ${ }^{8}$, Hiroaki Nozawa ${ }^{9}$, Hiroshi Matsumoto ${ }^{10}$, Kentaro Yamazaki ${ }^{11}$, Sae-Won Han ${ }^{12}$, Wei Wang ${ }^{13}$, Joong Bae Ahn ${ }^{14}$, Yanhong Deng ${ }^{15}$, Sang-Hee Cho ${ }^{16}$, Yi Ba ${ }^{17}$, Keun-Wook Lee ${ }^{18}$, Tao Zhang ${ }^{19}$, Taroh Satoh ${ }^{20}$, Marc E. Buyse ${ }^{21}$, Baek-Yeol Ryoo ${ }^{22}$, Lin Shen ${ }^{23}$, Junichi Sakamoto ${ }^{24}$ and Tae Won Kim² ${ }^{22^{*}}$

\begin{abstract}
Background: Capecitabine and irinotecan combination therapy (XELIRI) has been examined at various dose levels to treat metastatic colorectal cancer (mCRC). Recently, in the Association of Medical Oncology of the German Cancer Society (AIO) 0604 trial, tri-weekly XELIRI plus bevacizumab, with reduced doses of irinotecan $\left(200 \mathrm{mg} / \mathrm{m}^{2}\right.$ on day 1) and capecitabine $\left(1600 \mathrm{mg} / \mathrm{m}^{2}\right.$ on days 1-14), repeated every 3 weeks, has shown favorable tolerability and efficacy which were comparable to those of capecitabine and oxaliplatin (XELOX) plus bevacizumab. The doses of capecitabine and irinotecan in the AIO trial are considered optimal. In a phase I/II study, XELIRI plus bevacizumab (BIX) as second-line chemotherapy was well tolerated and had promising efficacy in Japanese patients.
\end{abstract}

Methods: The Asian XELIRI ProjecT (AXEPT) is an East Asian collaborative, open-labelled, randomized, phase III clinical trial which was designed to demonstrate the non-inferiority of XELIRI with or without bevacizumab versus standard FOLFIRI (5-fluorouracil, leucovorin, and irinotecan combination) with or without bevacizumab as second-line chemotherapy for patients with mCRC. Patients with 20 years of age or older, histologically confirmed mCRC, Eastern Cooperative Oncology Group performance status 0-2, adequate organ function, and disease progression or intolerance of the first-line regimen will be eligible. Patients will be randomized (1:1) to receive standard FOLFIRI with or without bevacizumab ( $5 \mathrm{mg} / \mathrm{kg}$ on day 1 ), repeated every 2 weeks (FOLIRI arm) or XELIRI with or without bevacizumab ( $7.5 \mathrm{mg} / \mathrm{kg}$ on day 1), repeated every 3 weeks (XELIRI arm). A total of 464 events were estimated as necessary to show non-inferiority with a power of $80 \%$ at a one-sided a of 0.025 , requiring a target sample size of 600 patients. The $95 \%$ confidence interval (CI) upper limit of the hazard ratio was pre-specified as less than 1.3.

\footnotetext{
*Correspondence: twkimmd@amc.seoul.kr

22 Department of Oncology, Asan Medical Center, University of Ulsan

Collage of Medicine, Seoul 138-736, South Korea

Full list of author information is available at the end of the article
} 
Conclusion: The Asian XELIRI ProjecT is a multinational phase III trial being conducted to provide evidence for XELIRI with or without bevacizumab as a second-line treatment option of mCRC.

Trial registration ClinicalTrials.gov NCT01996306. UMIN000012263

Keywords: Metastatic colorectal cancer, Randomized phase III clinical trial, XELIRI, Bevacizumab, Second-line therapy

\section{Background}

Life-prolonging systemic therapies, e.g., chemotherapies with or without molecular targeted agents such as anti-vascular endothelial growth factor (VEGF) or antiepidermal growth factor receptor (EGFR) agents, are important for unresectable metastatic colorectal cancer (mCRC). The National Comprehensive Cancer Network (NCCN) guidelines [1], the European Society for Medical Oncology (ESMO) clinical practice guidelines [2], and the Japanese Society for Cancer of the Colon and Rectum (JSCCR) guidelines [3] recommend four basic cytotoxic chemotherapy regimens as options to patients with $\mathrm{mCRC}$ who are able to tolerate intensive therapy.

Recently, head-to-head randomized phase III studies comparing bevacizumab and cetuximab (e.g., FIRE-3 and CALGB80405) did not show a consistent substantial difference in response rate, overall survival (OS), or progression-free survival (PFS) [4-6]. A randomized phase III study (STRATEGIC-1) that was designed to determine the best sequence of systemic therapy is now in progress [7].

Combination chemotherapy using oral drugs is convenient, freeing patients from chemoports or infusion pumps. However, compelling evidence for the safety and efficacy of such regimens is required.

In a phase III BICC-C study conducted mainly in the United States, tri-weekly XELIRI regimen (also named CapeIRI regimen: intravenous infusion of irinotecan $250 \mathrm{mg} / \mathrm{m}^{2}$ on day 1 and oral administration of capecitabine $2000 \mathrm{mg} / \mathrm{m}^{2}$ per day on days $\left.1-15\right)$ was compared with FOLFIRI regimen (intravenous infusion of irinotecan $180 \mathrm{mg} / \mathrm{m}^{2}$, leucovorin $[\mathrm{LV}] 400 \mathrm{mg} / \mathrm{m}^{2}$, and 5 -fluorouracil [5-FU] $400 \mathrm{mg} / \mathrm{m}^{2}$ on day 1 followed by a 46 -hour infusion of 5 -FU $2400 \mathrm{mg} / \mathrm{m}^{2}$, repeated every 2 weeks) and modified IFL regimen (intravenous infusion of irinotecan $125 \mathrm{mg} / \mathrm{m}^{2}$ on day $1, \mathrm{LV} 20 \mathrm{mg} / \mathrm{m}^{2}$ and $5-\mathrm{FU}$ $500 \mathrm{mg} / \mathrm{m}^{2}$ on days 1 and 8 , repeated every 3 weeks) [8]. Grade 3/4 adverse events mainly consisting of gastrointestinal toxicities occurred more frequently in patients treated with CapeIRI than in those treated with FOLFIRI (nausea, $18.4 \%$ vs. $8.8 \%$; diarrhea, $47.5 \%$ vs. $13.9 \%$; dehydration, $19.1 \%$ vs. $5.8 \%$ ); median PFS was significantly shorter for patients treated with CapeIRI than for those treated with FOLFIRI (5.8 vs. 7.6 months, $P=0.015$ ) due to early discontinuation of CapeIRI regimen. The authors suggested that the large number of patients with early treatment discontinuations for adverse events in the CapeIRI group may because of regional and ethnic differences in the metabolism of 5-FU and capecitabine, especially between patients in the United States and East Asia $[8,9]$. Subsequently, a modified XELIRI regimen, with reduced doses of irinotecan and capecitabine, was studied in combination with bevacizumab, mainly in studies comparing FOLFIRI and XELOX regimens (intravenous infusion of oxaliplatin $130 \mathrm{mg} / \mathrm{m}^{2}$ on day 1 plus oral administration of capecitabine $2000 \mathrm{mg} / \mathrm{m}^{2}$ per day on days 1-15) [10-13].

Recently, in the AIO 0604 trial, tri-weekly XELIRI plus bevacizumab, with reduced doses of irinotecan $(200 \mathrm{mg} /$ $\mathrm{m}^{2}$ on day 1$)$ and capecitabine $\left(1600 \mathrm{mg} / \mathrm{m}^{2}\right.$ per day on days 1-14), was compared with XELOX plus bevacizumab [13]. Common grade $3 / 4$ adverse events included diarrhea (16\% for the XELIRI arm and $22 \%$ for the XELOX arm), nausea (3\% for each arm), and fatigue (3\% for each arm). The median PFS was 12.1 vs. 10.4 months [13]. This randomized phase II trial showed that XELIRI plus bevacizumab had equivalent efficacy to XELOX plus bevacizumab, even though the XELIRI-based regimen was designed primarily to reduce adverse events.

In Japan, a completed phase I/II study evaluated the efficacy of the XELIRI regimen using the same dose in the AIO 0604 trial for patients with mCRC who had previously been treated with oxaliplatin and bevacizumab (the BIX study) [14]. The most common grade $3 / 4$ adverse events were neutropenia (8.8\%), nausea $(5.9 \%)$, diarrhea (5.9\%), and fatigue (2.9\%). The efficacy analysis demonstrated an overall response rate of $17.6 \%$ and median PFS of 8.3 months. These results suggest that XELIRI plus bevacizumab would be safe and effective for East Asian patients with mCRC. Considering that there are regional differences between the United States and East Asian patients with respect to the metabolism of capecitabine and 5-FU and that gastrointestinal toxicities may be more tolerable for East Asian patients, XELIRI plus bevacizumab may be more appropriate for Asian patients [9]. Therefore, XELIRI with or without bevacizumab was assigned to the study therapy group in the AXEPT trial.

Homozygosity or double heterozygosity for UDP-glucuronosyl transferase 1A1 (UGT1A1) polymorphisms (UGT1A1*28 and UGT1A1*6) may relate to increased 
serious adverse events, such as neutropenia and diarrhea, in patients treated with an irinotecan-based regimen. A reduced dose of irinotecan is therefore needed in patients with these polymorphisms [15-19].

\section{Methods \\ Objectives}

The primary objective is to demonstrate the non-inferiority in terms of OS for XELIRI with or without bevacizumab versus FOLFIRI with or without bevacizumab as second-line therapy for patients with mCRC.

The secondary objectives are to evaluate the PFS, time to treatment failure (TTF), overall response rate (ORR), disease control rate (DCR), relative dose intensity (RDI), safety, and association between $U G T 1 A 1$ polymorphisms and the occurrence rates of adverse events. Exploratory subgroup analysis is planned to investigate factors which are thought to influence prognosis, including country, Kirsten rat sarcoma viral oncogene homolog (KRAS) genotype, and UGT1A1 genotype.

XELIRI improves treatment convenience by eliminating continuous intravenous infusion and permitting fewer hospital visits (every 3 weeks). Therefore, demonstration of the non-inferiority of XELIRI with or without bevacizumab versus FOLFIRI with or without bevacizumab will provide new evidence for this novel treatment option as second-line therapy for $\mathrm{mCRC}$.

\section{Trial design}

AXEPT is an East Asian collaborative, open-labelled, randomized, non-inferiority, phase III clinical trial comparing the efficacy and safety of XELIRI with or without bevacizumab versus FOLFIRI with or without bevacizumab in patients with mCRC.

After written informed consent has been obtained, eligible patients will be randomized (1:1) to either the XELIRI arm or the FOLFIRI arm using minimization methods by the Swedish central electronic case-report form system (eCRF: VIEDOC ${ }^{\circledR}$, PCG Solutions Co. Ltd., Uppsala, Sweden). Stratification factors will include (1) country (Japan vs. South Korea vs. China), (2) Eastern Cooperative Oncology Group performance status (ECOG PS) (0-1 vs. 2), (3) number of metastatic sites (1 vs. >1), (4) prior oxaliplatin treatment (yes vs. no), and (5) concomitant bevacizumab treatment (with vs. without).

The study will be conducted in three countries, Japan, South Korea, and China (Fig. 1). The steering committee consists of a principal investigator from each country and a biostatistician. A total of 600 patients will be enrolled from 73 Japanese hospitals, 8 South Korean hospitals, and 17 Chinese hospitals.

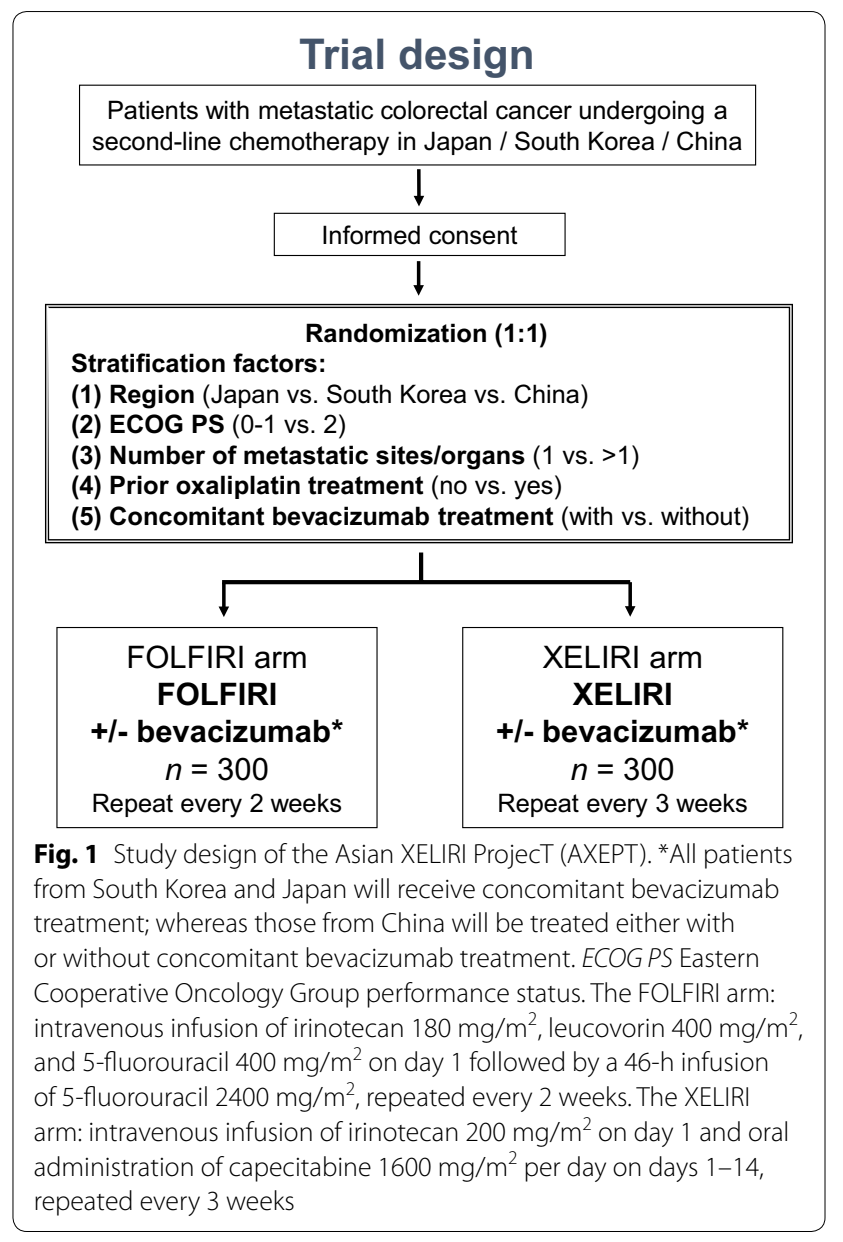

\section{Study population}

Patients who meet all of the below inclusion criteria and none of the exclusion criteria will be eligible for enrollment in this study.

\section{Inclusion criteria}

1. Histologically confirmed unresectable colorectal adenocarcinoma

2. Age $\geq 20$ years

3. ECOG PS 0-2

4. Signed and dated written informed consent

5. Life expectancy $>90$ days

6. Withdrawal from first-line chemotherapy (regardless of the combination with or without molecular targeted agents) for mCRC due to intolerable toxicity or disease progression, or relapse within 180 days after the last dose of adjuvant chemotherapy

7. Adequate organ functions according to the following laboratory values which are obtained within 14 days before enrollment 
- Neutrophil count $\geq 1500 / \mathrm{mm}^{3}$

- Platelet count $\geq 100 \times 10^{3} / \mathrm{mm}^{3}$

- Hemoglobin $\geq 9.0 \mathrm{~g} / \mathrm{dL}$

- Total bilirubin $\leq 1.5 \mathrm{mg} / \mathrm{dL}$

- Aspartate aminotransferase (AST) $\leq 100 \mathrm{IU} / \mathrm{L}$ (100 U/L) ( $\leq 200 \mathrm{IU} / \mathrm{L}$ [200 U/L] if liver metastases are present)

- Alanine transaminase (ALT) $\leq 100 \quad \mathrm{IU} / \mathrm{L}$ $(100 \mathrm{U} / \mathrm{L})(\leq 200 \mathrm{IU} / \mathrm{L}$ [200 U/L] if liver metastases are present)

- Serum creatinine $\leq 1.5 \mathrm{mg} / \mathrm{dL}$

\section{Exclusion criteria}

1. History of other malignancies with a disease-free interval $<5$ years

2. Massive pleural effusion or ascites requiring intervention

3. Radiological evidence of brain tumor or brain metastases

4. Active infection, including hepatitis

5. Any of the following concurrent diseases:

- Gastrointestinal bleeding or gastrointestinal obstruction (including paralytic ileus)

- Symptomatic heart disease (including unstable angina, myocardial infarction, and heart failure)

- Interstitial pneumonia or pulmonary fibrosis

- Uncontrolled diabetes mellitus

- Uncontrolled diarrhea (that interferes with daily activities despite adequate therapy)

6. Any of the following in the medical history:

- Myocardial infarction (history of one episode within 1 year before enrollment or two or more lifetime episodes)

- Serious hypersensitivity to any of the study drugs

- History of adverse reaction to fluoropyrimidines suggesting dihydropyrimidine dehydrogenase deficiency

7. Previous treatment with irinotecan

8. Current treatment with atazanavir sulfate

9. Previous treatment with tegafur, gimeracil, and oteracil potassium within 7 days before enrollment

10. Pregnant or lactating women, and men or women unwilling to use contraception

11. Requirement for continuous treatment with steroids

12. Psychiatric disability that would preclude study compliance

13. Otherwise determined by the investigator to be unsuitable for participation in the study
Additional exclusion criteria for those receiving bevacizumab:

1. Concurrent gastrointestinal perforation or history of gastrointestinal perforation within 1 year before enrollment

2. History of pulmonary hemorrhage/hemoptysis $\geq$ grade 2 (defined as at least $2.5 \mathrm{~mL}$ of bright red blood) within 1 month before enrollment

3. History of laparotomy, thoracotomy, or intestinal resection within 28 days before enrollment

4. Incomplete wound healing (except suture wounds from implantation of a central venous port), gastrointestinal ulcer, or traumatic fracture

5. Current or recent (within 1 year) thromboembolism or cerebrovascular disease

6. Currently receiving or requiring anticoagulation therapy (>325 mg/day of aspirin)

7. Bleeding diathesis, coagulopathy, or coagulation factor abnormality (international normalized ratio [INR] $\geq 1.5$ within 14 days before enrollment)

8. Uncontrolled hypertension

9. Urine protein by dipstick $>+2$

\section{Study treatment}

Patients will be randomly assigned to receive one of the following treatments:

The FOLFIRI arm: intravenous infusion of irinotecan $180 \mathrm{mg} / \mathrm{m}^{2}, l$-LV $200 \mathrm{mg} / \mathrm{m}^{2}$ (or $d, l-\mathrm{LV} 400 \mathrm{mg} / \mathrm{m}^{2}$ ), bevacizumab $5 \mathrm{mg} / \mathrm{kg}$, and 5 -FU $400 \mathrm{mg} / \mathrm{m}^{2}$ on day 1 followed by a 46 -hour continuous infusion of 5 -FU $2400 \mathrm{mg} / \mathrm{m}^{2}$, repeated every 2 weeks until disease progression, unacceptable toxicity, or patient withdrawal.

The XELIRI arm: intravenous infusion of irinotecan $200 \mathrm{mg} / \mathrm{m}^{2}$ and bevacizumab $7.5 \mathrm{mg} / \mathrm{kg}$ on day 1 , and oral administration of capecitabine $1600 \mathrm{mg} / \mathrm{m}^{2}$ on days 1-14, repeated every 3 weeks until disease progression, unacceptable toxicity, or patient withdrawal.

In both arms, the dose for irinotecan will be started at $150 \mathrm{mg} / \mathrm{m}^{2}$ in patients identified to be homozygous for UGT1A1*6 or UGT1A1*28 or double heterozygous for $U G T 1 A 1^{*} 6$ and $U G T 1 A 1 * 28$ at baseline screening [20].

All adverse events will be assessed according to the National Cancer Institute Common Toxicity Criteria Adverse Event v4.0 (NCI CTCAE v4.0) [21].

In both arms, the protocol treatment will be started upon the investigator's decision based on lab values within the inclusion criteria at the start of a treatment cycle. The next cycle should not be administered unless the neutrophil count $\geq 1500 / \mathrm{mm}^{3}$, platelet count $\geq 75,000 / \mathrm{mm}^{3}$, serum total bilirubin $\leq 1.5 \mathrm{mg} /$ $\mathrm{dL}$, serum creatinine $\leq 1.5 \mathrm{mg} / \mathrm{dL}$, hand-foot syndrome 
grade $\leq 1$, and other non-hematologic toxicities resolve to grade $\leq 1$. In those receiving bevacizumab, treatment should not be administered unless hypertension grade $\leq 2$, proteinuria $\leq 2+$, venous thromboembolism grade $\leq 2$, and hemorrhage grade $\leq 1$. If the next cycle cannot be started within 28 days of the scheduled start date, the protocol treatment will be discontinued. If adverse events that require dose reduction occur prior to a cycle, dose reduction could be done twice as appropriate. If more than two dose reductions are required, treatment with that drug will be discontinued. Once a dose reduction has been done, the dose should not be increased in subsequent cycles.

\section{Statistical considerations}

The analysis set is the all-randomized population. In the primary analysis, the cumulative OS curve, median OS, and annual OS rate will be estimated with the KaplanMeier method, and the confidence interval (CI) of the median OS will be calculated with the Brookmeyer and Crowley method. The point estimates of the hazard ratio (HRs) and their 95\% CIs will be calculated with a Cox proportional hazard model.

Secondary endpoints will be analyzed to supplement the results of the primary analysis. No adjustments will be made for multiplicity because the analysis of secondary endpoints is exploratory. Intergroup comparisons will be performed as necessary, but $P$ values obtained from statistical tests will be considered reference data only. Exploratory subgroup analysis for factors thought to influence prognosis, including country, KRAS genotype, and $U G T 1 A 1$ genotype, will be performed.

The sample size of this study was calculated on the basis of two previously reported phase III studies which included FOLFIRI plus bevacizumab or XELIRI plus bevacizumab as second-line treatment of patients with mCRC. In the FIRIS study, the median survival time (MST) was 18.2 months in the FOLFIRI group (13.7 months in patients previously treated with oxaliplatin-based therapy) [22]. In the ML18147 study, the MST was 10.0 months in the group receiving irinotecanbased chemotherapy, such as FOLFIRI and XELIRI, and 12.0 months in the group receiving irinotecan-based chemotherapy plus bevacizumab [23]. The add-on effect of bevacizumab has also been confirmed by phase II studies on Japanese [24], South Korean [25, 26], and Chinese patients $[27,28]$ and by a retrospective analysis report [29]. In addition, the results of the ML18147 trial [23] and E3200 trial [30] indicate that the add-on effect of bevacizumab in second-line treatment is similar between patients with and without previous treatment of concomitant bevacizumab. Based on the above considerations, we assumed to observe an MST of 11.0 months for patients treated with FOLFIRI and 13.0 months for patients treated with FOLFIRI plus bevacizumab. Due to differences in regional medical environment, all South Korean and Japanese patients will receive concomitant bevacizumab treatment, whereas patients enrolled from China are expected to include those who do not receive concomitant bevacizumab treatment. On the basis of the estimated proportion of patients receiving treatment with or without bevacizumab and the estimated MST for each of these groups, the MST of patients in the FOLFIRI arm was assumed to be 12.6 months. Calculation of the required sample size under these conditions with the following assumptions revealed that an estimated 464 events would be needed to achieve at least $80 \%$ power.

(i) $\mathrm{HR}$ of treatment to control: 1.00 (MST, 12.6 months for the FOLFIRI arm vs. 12.6 months for the XELIRI arm)

(ii) Upper margin of the non-inferiority hypothesis: HR of 1.30 (MST, 12.6 months for the FOLFIRI arm vs. 9.7 months for the XELIRI arm)

(iii) One-sided significance level: 0.025

(iv) Enrollment period: 24 months

(v) Follow-up period: 18 months

An enrollment of 600 patients, including a 5\% annual dropout rate, was therefore set in the study.

The non-inferiority upper margin of HR was set at 1.30 (9.7 months as converted to MST) considering the variation of the $95 \%$ CI of MST with stratification by KRAS status or therapy with anti-EGFR antibody drugs after the protocol treatment. If the above non-inferiority hypothesis was achieved, the hypothesis will be tested using a non-inferiority upper margin of HR of 1.25.

For sensitivity analysis, Cox regression analysis will also be performed, adding the KRAS status as a covariate to avoid the influence of anti-EGFR antibody treatment after the protocol treatment. If necessary, Cox regression analysis will be performed with adjusted demographic factors (for imbalance between the two treatment arms) rather than stratification factors.

\section{Study coordination and ethical aspects}

The study will be conducted according to the protocol in compliance with the principles of the Declaration of Helsinki, International Conference of Harmonization Good Clinical Practice Efficacy 6 (ICH-GCP E6) [31], and the rules and regulations of each country.

The Epidemiological and Clinical Research Information Network (ECRIN) is responsible for study management (including enrollment) and monitoring of Japanese sites and will also assist with and oversee local study management in each data center. ECRIN delegates its 
responsibility to the South Korean and Chinese local data centers for study preparation, contract, patient enrollment within the designated study term, exchange of safety data, document archives, quality checks/quality assurance, and other local procedures which are stated in the ICH-GCP and local regulations.

The protocol and the informed consent form used in the study must be approved by the Institutional Review Board (IRB)/Independent Ethics Committee (IEC) at each study site prior to the start of study. If IRB/IEC approval is obtained, the site principal investigator will send the copy of IRB/IEC approval document to each data center. The original IRB/IEC approval document will be retained by the site principal investigator, and a copy will be retained at the local data center.

The study protocol was approved by the ECRIN central IRB on September 3, 2013 and was registered at Clinicaltrials.gov (NCT01996306 on November 22, 2013) and UMIN-CTR (UMIN000012263 on November 11, 2013).

In this study, an Independent Data Monitoring Committee (IDMC) is established to determine whether this study is conducted appropriately. The role of the IDMC is to assess at intervals the progress of a clinical trial, the safety data, and the critical efficacy endpoints and to recommend the Steering Committee and study sponsor whether to continue, modify, or stop the trial. IDMC members will not be directly involved in the conduction or operation of the trial.

\section{Discussion}

The XELIRI regimen has already been examined at various doses and combinations since the beginning of the twenty first century $[8,10-14,25]$. The XELIRI regimen used in the AIO 0604 trial is regarded appropriate in terms of efficacy and safety [13]. In addition, according to the ML18147 study [23], 12\% of all enrolled patients were treated with the tri-weekly XELIRI plus bevacizumab regimen (AIO XELIRI regimen), and approximately 35\% with the irinotecan-based regimen.

However, XELIRI has not been recommended by guidelines (neither from ESMO nor NCCN) because of its toxicities. For that reason, a phase II study (the BIX trial) was conducted to determine the tolerability of the AIO XELIRI regimen in Japanese patients. The results showed that the safety profile was acceptable and the efficacy was promising [14]. Thus, we planned this randomized phase III trial, collaborating with investigators from China and South Korea.

In addition, with regard to $U G T 1 A 1$ polymorphisms, it is necessary to evaluate the association between UGT1A1 polymorphisms and safety or efficacy in East Asian population and to establish clear rules of dose reduction for irinotecan. Thus, we will check UGT1A1 genotype at the baseline screening and set an initial irinotecan dose for patient with UGT1A1 polymorphism. The association between UGT1A1 genotype and safety will be further explored in subgroup analysis.

Demonstration of the non-inferiority of XELIRI with or without bevacizumab to FOLFIRI with or without bevacizumab in our study will provide evidence for a new treatment option as second-line therapy for $\mathrm{mCRC}$.

\section{Authors' contributions}

$\mathrm{KM}, \mathrm{YP}, \mathrm{TK}$, and $\mathrm{RX}$ are responsible for the trial design and are the principal investigators. SM is the statistician. $\mathrm{Sl}$ is the study secretary, coordinating study management. HU, TN, HN, HM, SH, WW, JA, YD, SC, YB, KL, TZ, and KY are participants of protocol coordination committee in all phases of this study, including the design and draft of this protocol. MK, JS, and RX prepared the manuscript. SH, WW, JA, YD, SC, YB, KL, and TZ are study coordinators in each country. JS as a study sponsor is responsible for study protocol, data collection, data analysis, and preparation of publication. All authors read and approved the final manuscript.

\section{Author details}

${ }^{1}$ Gastrointestinal Cancer Center, Sano Hospital, Hyogo 655-0031, Japan. ${ }^{2}$ State Key Laboratory of Oncology in South China, Department of Medical Oncology, Collaborative Innovation Center for Cancer Medicine, Sun Yat-sen University Cancer Center, Guangzhou, Guangdong 510060, P. R. China. ${ }^{3}$ Department of Clinical Oncology, Aichi Cancer Center Hospital, Nagoya 464-8681, Japan. ${ }^{4}$ Department of Medicine, Samsung Medical Center, Sungkyunkwan University School of Medicine, Seoul 135-710, South Korea. ${ }^{5}$ Department of Biomedical Statistics and Bioinformatics, Graduate School of Medicine, Kyoto University, Kyoto 606-8501, Japan. ${ }^{6}$ Gastrointestinal Medical Oncology Division, National Cancer Center Hospital, Tokyo 104-0045, Japan. ${ }^{7}$ Department of Surgical Specialties, Graduate School, Tokyo Medical and Dental University, Tokyo 113-8519, Japan. ${ }^{8}$ Department of Gastrointestinal Medical Oncology, National Hospital Organization Shikoku Cancer Center, Matsuyama 791-0280, Japan. ${ }^{9}$ Department of Surgical Oncology, The University of Tokyo, Tokyo 113-0033, Japan. ${ }^{10}$ Department of Surgery, Tokyo Metropolitan Komagome Hospital, Tokyo 113-8677, Japan. ${ }^{11}$ Division of Gastrointestinal Oncology, Shizuoka Cancer Center, Shizuoka 411-8777, Japan. ${ }^{12}$ Department of Internal Medicine, Seoul National University Hospital, Seoul 110-744, South Korea. ${ }^{13}$ Department of Gastrointestinal Oncology, The First People's Hospital of Foshan, Foshan, Guangdong 528000, P. R. China. ${ }^{14}$ Department of Internal Medicine, Yonsei University College of Medicine, Seoul 120-752, South Korea. ${ }^{15}$ Department of Medical Oncology, The Sixth Affiliated Hospital, Sun Yat-sen University, Guangzhou, Guangdong 510655, P. R. China. ${ }^{16}$ Department of Hematology-Oncology, Chonnam National University Medical School, Gwangju 519-809, South Korea. ${ }^{17}$ Department of Digestive Oncology, Tianjin Medical University Cancer Institute and Hospital, Tianjin 300060, P. R. China.

${ }^{18}$ Department of Internal Medicine, Seoul National University Bundang HospitalSeoul National University College of Medicine, Seongnam 463-707, South Korea. ${ }^{19}$ Department of Medical Oncology, Union Hospital of Tongji Medical College, Huazhong University of Science and Technology, Wuhan, Hubei 430022, P. R. China. ${ }^{20}$ Department of Gastroenterological Surgery, Graduate School of Medicine, Osaka University, Osaka 565-0871, Japan. ${ }^{21}$ International Drug Development Institute, Louvain-La-Neuve 1340, Belgium. ${ }^{22}$ Department of Oncology, Asan Medical Center, University of Ulsan Collage of Medicine, Seoul 138-736, South Korea. ${ }^{23}$ Department of Gastrointestinal Oncology, Peking University Cancer Hospital \& Institute, Beijing 100-142, P. R. China. ${ }^{24}$ Tokai Central Hospital, Kakamigahara 504-8601, Japan.

\section{Acknowledgements}

This trial is supported by Epidemiological and Clinical Research Information Network (ECRIN: global sponsor), Asan Medical Center Academic-Research office, and Sun Yat-sen University, and funded by Chugai Pharmaceutical Co., Ltd. and F. Hoffmann-La Roche Ltd.

\section{Competing interests}

MK gets honoraria from Chugai Pharmaceutical Co., Ltd, Takeda Pharmaceutical Co., Ltd., Yakult Honsha Co., Ltd., and Merck Serono Co., Ltd. SM gets 
honoraria from Chugai Pharmaceutical Co., Ltd and Daiichi Sankyo Co., Ltd. as well as research funding from Chugai Pharmaceutical Co., Ltd. YK gets honoraria from Chugai Pharmaceutical Co., Ltd, Takeda Pharmaceutical Co., Ltd., Yakult Honsha Co., Ltd., Merck Serono Co., Ltd., Bayer AG, Bristol-Myers Squibb K.K, and Taiho Pharmaceutical Co., Ltd. as well as research funding from BristolMyers Squibb K.K. Sl gets honoraria from Chugai Pharmaceutical Co., Ltd. as well as research funding from Chugai Pharmaceutical Co., Ltd., Daiichi Sankyo Co., Ltd., and Kyowa Kirin Co., Ltd. TK gets honoraria from Amgen, Eli Lilly \& Co as well as research funding from Merck Serono Co., Ltd. and Bayer AG, F. Hoffmann-La Roche Ltd. JS is a consultant and advisor for Takeda Pharmaceutical Co., Ltd. and gets honoraria from Tsumura \& Co. TN gets honoraria from Chugai Pharmaceutical Co., Ltd., Yakult Honsha Co., Ltd., Takeda Pharmaceutical Co., Ltd., Taiho Pharmaceutical Co., Ltd., and Bayer AG, Eli Lilly Japan K.K. KM gets honoraria from Chugai Pharmaceutical Co., Ltd. and Yakult Honsha Co., Ltd. TS is a consultant and advisor for Bayer AG and Eli Lilly Japan K.K.; gets honoraria from Chugai Pharmaceutical Co., Ltd., Merck Serono Co., Ltd., BristolMyers Squibb K.K, and Takeda Pharmaceutical Co., Ltd.; and gets research funding from Chugai Pharmaceutical Co., Ltd. All authors declared that they have no competing interests.

\section{Funding}

This study has received funding from Chugai Pharmaceutical Co.Ltd., Roche Korea Co. Ltd., and Roche Shanghai. Co. Ltd.

Received: 21 July 2016 Accepted: 4 September 2016

Published online: 22 December 2016

\section{References}

1. National comprehensive cancer network clinical practice guidelines in oncology colon cancer Version 3. 2015. http://www.nccn.org/professionals/physician_gls/pdf/colon.pdf. Accessed 6 Aug 2015.

2. Van Cutsem E, Cervantes A, Nordlinger B, Arnold D, ESMO Guidelines Working Group, et al. Metastatic colorectal cancer: ESMO clinical practice guidelines for diagnosis, treatment and follow-up. Ann Oncol. 2014:25(Suppl 3):1-9.

3. Japanese society for cancer of the colon and rectum: JSCCR Guidelines 2014 for the treatment of colorectal cancer. Tokyo: Kanehara \& Co., Ltd; 2010.

4. Heinemann V, von Weikersthal LF, Decker T, Kiani A, Vehling-Kaiser U, Al-Batran SE, et al. FOLFIRI plus cetuximab versus FOLFIRI plus bevacizumab as first-line treatment for patients with metastatic colorectal cancer (FIRE-3): a randomised, open-label, phase 3 trial. Lancet Oncol. 2014;10(15):1065-75.

5. Venook AP, Niedzwiecki D, Lenz H, Innocenti F, Mahoney MR, O'Neil BH, et al. CALGB/SWOG 80405: phase III trial of irinotecan/5-FU/leucovorin (FOLFIRI) or oxaliplatin/5-FU/leucovorin (mFOLFOX6) with bevacizumab (BV) or cetuximab (CET) for patients (pts) with KRAS wild-type (wt) untreated metastatic adenocarcinoma of the colon or rectum (MCRC). J Clin Oncol. 2014;32(15):3

6. Mellas N, Benbrahim Z, El Mesbahi O. Colorectal cancer: new developments after the 2013 ECCO/ESMO congress. Chin J Cancer. 2014;33(4):218-21.

7. Chibaudel B, Bonnetain F, Tournigand C, de Larauze MH, de Gramont A, Laurent-Puig P, et al. STRATEGIC-1: a multiple-lines, randomized, openlabel GERCOR phase III study in patients with unresectable wild-type RAS metastatic colorectal cancer. BMC Cancer. 2015;15:496-509.

8. Fuchs CS, Marshall J, Mitchell E, Wierzbicki R, Ganju V, Jeffery M, et al. Randomized, controlled trial of irinotecan plus infusional, bolus, or oral fluoropyrimidines in first-line treatment of metastatic colorectal cancer: results from the BICC-C Study. J Clin Oncol. 2007;25(30):4779-86.

9. Haller DG, Cassidy J, Clarke SJ, Cunningham D, Van Cutsem E, Hoff PM et al. Potential regional differences for the tolerability profiles of fluoropyrimidines. J Clin Oncol. 2008:26(13):2118-23.

10. Souglakos J, Ziras N, Kakolyris S, Boukovinas I, Kentepozidis N, Makranto-

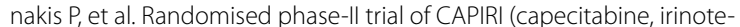
can) plus bevacizumab vs FOLFIRI (folinic acid, 5-fluorouracil, irinotecan) plus bevacizumab as first-line treatment of patients with unresectable/ metastatic colorectal cancer (mCRC). Br J Cancer. 2012;106(3):453-9.
11. Pectasides D, Papaxoinis G, Kalogeras KT, Eleftheraki AG, Xanthakis I, Makatsoris T, et al. XELIRI-bevacizumab versus FOLFIRI-bevacizumab as first-line treatment in patients with metastatic colorectal cancer: a Hellenic Cooperative Oncology Group phase III trial with collateral biomarker analysis. BMC Cancer. 2012;12:271-82.

12. Ducreux M, Adenis A, Pignon JP, François E, Chauffert B, Ichanté JL,

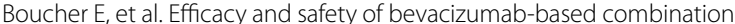
regimens in patients with previously untreated metastatic colorectal cancer: final results from a randomised phase II study of bevacizumab plus 5-fluorouracil, leucovorin plus irinotecan versus bevacizumab plus capecitabine plus irinotecan (FNCLCC ACCORD 13/0503 study). Eur J Cancer. 2013;49(6):1236-45.

13. Schmiegel W, Reinacher-Schick A, Arnold D, Kubicka S, Freier W, Dietrich $\mathrm{G}$, et al. Capecitabine/irinotecan or capecitabine/oxaliplatin in combination with bevacizumab is effective and safe as first-line therapy for metastatic colorectal cancer: a randomized phase II study of the AIO colorectal study group. Ann Oncol. 2013;24(6):1580-7.

14. Hamamoto Y, Yamaguchi T, Nishina T, Yamazaki K, Ura T, Nakajima T, et al. A phase I/II study of XELIRI plus bevacizumab as second-line chemotherapy for Japanese patients with metastatic colorectal cancer (BIX study). Oncologist. 2014;19(11):1131-2.

15. Beutler E, Gelbart T, Demina A. Racial variability in the UDP-glucuronosyltransferase 1 (UGT1A1) promoter: a balanced polymorphism for regulation of bilirubin metabolism. Proc Natl Acad Sci. 1998;95(14):8170-4.

16. Hoskins Janelle M, Goldberg Richard M, Pingping Qu. UGT1A1*28 genotype and irinotecan-induced neutropenia: dose matters. J Natl Cancer Inst. 2007;99(17):1290-5.

17. Sai K, Saito Y, Sakamoto H, Shirao K, Kurose K, Saeki M, et al. Importance of UDP-glucuronosyltransferase $1 \mathrm{~A} 1 * 6$ for irinotecan toxicities in Japanese cancer patients. Cancer Lett. 2008;261(2):165-71.

18. Takano M, Kato M, Yoshikawa T, Sasaki N, Hirata J, Furuya K, et al. Clinical significance of UDP-glucuronosyl transferase $1 \mathrm{~A} 1 * 6$ for toxicities of combination chemotherapy with irinotecan and cisplatin in gynecologic cancers: a prospective multi-institutional study. Oncology. 2009;76(5):315-21

19. O'Dwyer PJ, Catalano RB. Uridine diphosphate glucuronosyltransferase (UGT) $1 \mathrm{~A} 1$ and irinotecan: practical pharmacogenomics arrives in cancer therapy. J Clin Oncol. 2006;24(28):4534-8.

20. Kim KP, Hong YS, Lee JL, Bae KS, Kim HS, Shin JG, et al. A phase I study of UGT1A1 ${ }^{*} 28 /{ }^{*} 6$ genotype-directed dosing of irinotecan (CPT-11) in Korean patients with metastatic colorectal cancer receiving FOLFIRI. Oncology. 2015;88(3):164-72.

21. National Cancer Institute, Cancer therapy evaluation program. common terminology criteria for adverse events (CTCAE). Version 4.0. http://evs.nci. nih.gov/ftp1/CTCAE/CTCAE_4.03_2010-06-14_QuickReference_5x7.pdf. Accessed 6 Aug 2015

22. Muro K, Boku N, Shimada Y. Irinotecan plus S-1 (IRIS) versus fluorouracil and folinic acid plus irinotecan (FOLFIRI) as second- line chemotherapy for metastatic colorectal cancer: a randomised phase 2/3 non-inferiority study (FIRIS study). Lancet Oncol. 2010;11(9):853-60.

23. Bennouna J, Sastre J, Arnold D, Österlund P, Greil R, Van Cutsem E, et al. Continuation of bevacizumab after first progression in metastatic colorectal cancer (ML18147): a randomised phase 3 trial. Lancet Oncol. 2013;14(1):29-37.

24. Nakayama G, Uehara K, Ishigure K, Yokoyama H, Ishiyama A, Eguchi $T$, et al. The efficacy and safety of bevacizumab beyond first progression in patients treated with first-line mFOLFOX6 followed by second-line FOLFIRI in advanced colorectal cancer: a multicenter, single-arm, phase II trial (CCOG-0801). Cancer Chemother Pharmacol. 2012;70(4):575-81.

25. Shin SJ, Ahn JB, Choi HJ, Cho BC, Jeung HC, Rha SY, et al. The combination of capecitabine and irinotecan in treating 5-Fluorouracil- and Oxaliplatinpretreated metastatic colorectal cancer. Cancer Chemother Pharmacol. 2008;61(1):75-81

26. Hong YS, Lee J, Kim KP, Lee JL, Park YS, Park JO, et al. Multicenter phase II study of second-line bevacizumab plus doublet combination chemotherapy in patients with metastatic colorectal cancer progressed after upfront bevacizumab plus doublet combination chemotherapy. Invest New Drugs. 2013;31(1):183-91. 
27. Bao HY, Fang WJ, Zhang XC, Shi GM, Huang S, Yu LF, et al. Phase II study of FOLFIRI regimen in patients with advanced colorectal cancer refractory to fluoropyrimidine and oxaliplatin. Cancer Chemother Pharmacol. 2011;67(1):147-52.

28. Cui F, Chen JZ, Wan C, Chen B, Luo RC, Zheng H. Clinical research of bevacizumab in combination with irinotecan, fluorouracil and leucovorin for advanced metastatic colorectal cancer. Zhonghua Wei Chang Wai Ke Za Zhi. 2009;12(4):374-7.

29. Zhou JF, Bai CM, Cheng YJ, Jia N, Shao YJ, Chen SC. Efficacy and safety of combination of irinotecan and capecitabine in patients with metastatic colorectal cancer after failure of chemotherapy with oxaliplatin. Zhongguo Yi Xue Ke Xue Yuan Xue Bao. 2009;31(3):358-61.
30. Giantonio BJ, Catalano PJ, Meropol NJ, O'Dwyer PJ, Mitchell EP, Alberts SR, Schwartz MA, et al. Bevacizumab in combination with oxaliplatin, fluorouracil, and leucovorin (FOLFOX4) for previously treated metastatic colorectal cancer: results from the Eastern Cooperative Oncology Group Study E3200. J Clin Oncol. 2007;25(12):1539-44.

31. International conference on harmonization of technical requirements for registration of pharmaceutical for human use, $\mathrm{ICH}$ harmonized tripartite guideline for good clinical practice, efficacy 6, http://www.ich. org/fileadmin/Public_Web_Site/ICH_Products/Guidelines/Efficacy/E6/ E6_R1_Guideline.pdf. Accessed 8 Aug 2016.

\section{Submit your next manuscript to BioMed Central and we will help you at every step:}

- We accept pre-submission inquiries

- Our selector tool helps you to find the most relevant journal

- We provide round the clock customer support

- Convenient online submission

- Thorough peer review

- Inclusion in PubMed and all major indexing services

- Maximum visibility for your research

Submit your manuscript at www.biomedcentral com/submit 